\title{
Correction to: The enhanced element enrichment in the supercritical states of granite-pegmatite systems
}

\author{
Rainer Thomas ${ }^{1} \cdot$ Paul Davidson $^{2}$ (I) $\cdot$ Karen Appel $^{3}$
}

Published online: 16 March 2019

(C) Science Press and Institute of Geochemistry, CAS and Springer-Verlag GmbH Germany, part of Springer Nature 2019

\section{Correction to: Acta Geochim} https://doi.org/10.1007/s11631-019-00319-z

In the original publication, there are some missing data in Table 1 and incorrect data in Table 2.

The correct data in Table 1 for Striegau/Strzegom, Poland, and the correct data in Table 2 for fitting parameter B2 in Granites, validated are provided in this correction article.
In the second paragraph in subsection 2.2 in the sixth line starting with "Ehrenfriedersdorf pegmatite system...", the text inside brackets should be read as see Fig. 7 (not as see Fig. 6a, b); also, in the fourth paragraph in the same section, the first line starting with "The formation of inclusions such as shown in ..." should be read as Fig. 7 not as $7 \mathrm{a}, \mathrm{b}$.

The original article can be found online at https://doi.org/10.1007/ s11631-019-00319-z.

Paul Davidson

Paul.Davidson@utas.edu.au

1 Section 3.6. Chemistry and Physics of Earth Materials, Helmholtz-Centre Potsdam, German Research Centre for Geoscience - GFZ, Telegrafenberg, 14473 Potsdam, Germany

2 CODES, Centre for Ore Deposit and Earth Sciences, School of Natural Sciences, University of Tasmania, Hobart, Australia

3 High Energy Density scientific instrument, European X-Ray Free-Electron Laser Facility GmbH, Holzkoppel 4, D-22869 Schenefeld, Germany 
Table 1 Experimental base: solvus data for pegmatites and granites, obtained from melt inclusions in quartz (and topaz) using the rapid quench cold-seal pressure vessel technique

\begin{tabular}{|c|c|c|c|c|c|}
\hline Pegmatites/granites & $\mathrm{T}_{\text {crit }}\left({ }^{\circ} \mathrm{C}\right)$ & $\mathrm{H}_{2} \mathrm{O}_{\text {crit }}(\%)$ & T-range $\left({ }^{\circ} \mathrm{C}\right)$ & Run pressure & Number of experiments \\
\hline \multicolumn{6}{|l|}{ Pegmatites } \\
\hline Amis Complex, Namibia & 700 & 29.2 & $550-700$ & $1-3$ & 6 \\
\hline Capoeira, Borborema, Brazil & 682 & 25.8 & $620-700$ & 3 & 7 \\
\hline Ehrenfriedersdorf, E-Germany & 718 & 23.6 & $500-750$ & $1-3$ & 16 \\
\hline Elba, Italy & 718 & 30.9 & $525-725$ & $1-3$ & 5 \\
\hline Hilbersdorf, E-Germany & 705 & 28.5 & $650-750$ & $1-3$ & 5 \\
\hline Malkhan pegmatite field, Russia & 721 & 27.6 & $600-750$ & $1-3$ & 14 \\
\hline Rito del Medio Pluton, N-New Mexico (USA) & 710 & 26.6 & $650-710$ & 3 & 4 \\
\hline Striegau/Strzegom, Poland & 699 & 26.4 & $600-700$ & $1-3$ & 4 \\
\hline Tanco, Canada & 762 & 27.5 & $600-750$ & $1-3$ & 8 \\
\hline Volyn, Ukraine & 732 & 27.4 & $650-750$ & 3 & 4 \\
\hline \multicolumn{6}{|l|}{ Granites } \\
\hline Eldzhurtinsk, Caucasus, Russia & 750 & 31.7 & $600-750$ & $1-3$ & 5 \\
\hline Hub Stock, topaz-albite-granite, Tschechien & 841 & 27.4 & $550-850^{\mathrm{a}}$ & $1-7.5$ & 15 \\
\hline Kirchberg Massif, E-Germany & 750 & 24.0 & $500-750^{\mathrm{a}}$ & $2-7.5$ & 25 \\
\hline Königshain Massif, E-Germany & 750 & 26.4 & $650-750$ & $1-3$ & 6 \\
\hline Zinnwald, topaz-albite-granite, E-Germany & 705 & 27.3 & $600-850^{\mathrm{b}}$ & $1-3$ & 8 \\
\hline
\end{tabular}

${ }^{a}$ Including high-pressure quenching experiments using a double-stage gas (He) chamber

${ }^{\mathrm{b}} \mathrm{Hydrothermal} \mathrm{rapid-quench}$ homogenization experiments at $850{ }^{\circ} \mathrm{C}$

Table 2 Fitting parameter for the solvus curves in normalized coordinates

\begin{tabular}{|c|c|c|c|c|c|}
\hline \multicolumn{6}{|c|}{ Equation: $\mathrm{Y}=\mathrm{A}+\mathrm{B} 1 * \mathrm{X}+\mathrm{B} 2 * \mathrm{X}^{2}+\mathrm{B} 3 * \mathrm{X}^{3}$} \\
\hline A & B1 & B2 & B3 & $\mathrm{R}$ & SD \\
\hline \multicolumn{6}{|l|}{ Granites } \\
\hline 0.84486 & 0.232 & -0.04202 & -0.0404 & 0.81 & 0.02587 \\
\hline \multicolumn{6}{|c|}{ Granites, validated } \\
\hline 0.8328 & 0.33082 & -0.16507 & $2.055 \times 10^{-5}$ & 0.9333 & 0.0141 \\
\hline \multicolumn{6}{|c|}{ Topaz-albite granite F3 } \\
\hline 0.50291 & 0.16782 & 1.48986 & -1.16455 & 0.9978 & 0.00597 \\
\hline \multicolumn{6}{|l|}{ Pegmatites } \\
\hline 0.76463 & 0.52904 & -0.35804 & 0.05694 & 0.9390 & 0.02176 \\
\hline
\end{tabular}

$\mathrm{Y}=(\mathrm{t}+273.15) /\left(\mathrm{t}_{\text {crit }}+273.15\right), \mathrm{t}$ in ${ }^{\circ} \mathrm{C}$

$\mathrm{X}=\mathrm{H}_{2} \mathrm{O} / \mathrm{H}_{2} \mathrm{O}_{\text {crit }}, \mathrm{H}_{2} \mathrm{O}$ in $[\%(\mathrm{~g} / \mathrm{g})], \mathrm{R}$ - correlation coefficient, SD-standard deviation 